\title{
Approximate Solutions of Schrödinger Equation under Manning-Rosen Potential in Arbitrary Dimension via SUSYQM
}

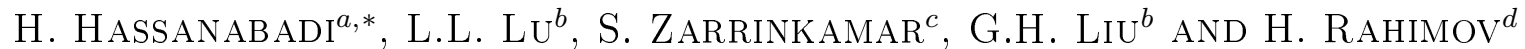 \\ ${ }^{a}$ Physics Department, Shahrood University of Technology, Shahrood, Iran \\ ${ }^{b}$ Department of Physics, College of Physics and Electronic Engineering, Guangzhou University \\ Guangzhou 510006, China \\ ${ }^{c}$ Department of Basic Sciences, Garmsar Branch, Islamic Azad University, Garmsar, Iran \\ ${ }^{d}$ Computer Engineering Department, Shahrood University of Technology, Shahrood, Iran
}

(Received September 16, 2011; revised version June 12, 2012; in final form June 21, 2012)

\begin{abstract}
The Schrödinger equation under the Manning-Rosen potential is solved in arbitrary dimension via the quantum mechanical idea of supersymmetry. The Pekeris approximation is used to overcome the inconsistency of the potential with the centrifugal term. Comments on the energy eigenvalue behavior versus dimension are included. The inter-dimensional degeneracy for various orbital quantum number $l$ and dimensions $D$ are studied. The expectation values of some physical parameters are reported via the Feynman-Hellmann theorem.
\end{abstract}

PACS: 03.65.Ge, 02.30.Gp, 03.65.-w, 34.20.Cf

\section{Introduction}

The rather old nonrelativistic Schrödinger equation appears as an either pedagogical or research background. Despite the long history of the field, there is still continuing interest in finding the corresponding solutions and exploring related consequences in physical sciences. Apart from the numerical programming, there are many exact and approximate analytical techniques including the Nikiforov-Uvarov (NU) [1, 2], supersymmetry quantum mechanics (SUSYQM) [3, 4], point canonical transformation (PCT) [3, 4], Lie algebraic [5], asymptotic iteration [6], shifted $1 / N$ expansion [7], exact quantization rule [8], the Hartree method [9], perturbation theory [10] and the ansatz approaches [11-13].

Our purpose of study is to solve the Schrödinger equation under the Manning-Rosen potential (MRP) which is a central interaction [14]. The central potentials are present in all branches of physics. In particle and high-energy physics, they describe the quark interactions [15]. In nuclear physics, they yield outstanding results in spectroscopy [16]. In atomic physics, they well describe the binding energy and inclusive momentum distributions $[17,18]$. In molecular physics, they have been used to investigate the intramolecular and intermolecular interactions and atomic pair correlation functions [18, 19]. Our focus in the present study is the Manning-Rosen potential [20], which yields outstanding phenomenological results. In particular, it has been applied to study the vibration modes diatomic molecules [21-23]. On the other hand, this interaction is the more general case of the sound Hulthén potential which itself yields motivating results in various fields such as nuclear and particle [24],

* corresponding author; e-mail: h.hasanabadi@shahroodut.ac.ir atomic [25], molecular [26] and chemical physics [27]. The MRP has been already studied under the Dirac [28], Klein-Gordon [29] and Schrodinger equation [30] by various analytical tools. Here, bearing in mind the deeper physical insight that analytical methodologies provide into the physics of the problem, we use the powerful SUSYQM in our calculations. For the sake of generality, we do our study in the $D$-dimensions. To be able to use the SUSYQM, we first apply a Pekeris-type approximation $[31,32]$ to the centrifugal term. Having calculated the approximate analytical solutions, we calculate some useful expectation values which are frequently present in various physical researches.

\section{The $D$-dimensional radial Schrödinger equation}

This potential possesses the form [20]:

$$
V(r)=\frac{V_{1}}{\left(\mathrm{e}^{\alpha r}-1\right)^{2}}+\frac{V_{2}}{\mathrm{e}^{\alpha r}-1},
$$

with

$$
\begin{aligned}
& V_{1}=\frac{\hbar^{2} \alpha^{2}\left[\alpha^{\prime}\left(\alpha^{\prime}-1\right)\right]}{2 \mu}, \\
& V_{2}=-\frac{\hbar^{2} b \alpha^{2}}{2 \mu},
\end{aligned}
$$

where $b$ and $\alpha^{\prime}$ are two constants and the parameter $\alpha$ characterizes the rang of the potential.

In Fig. 1 we have plotted the potential versus $r$. In the $D$-dimensional Hilbert space, the radial wave function $R_{n, l}(r)$ is [5]:

$$
\begin{aligned}
& {\left[\frac{\mathrm{d}^{2}}{\mathrm{~d} r^{2}}+\frac{D-1}{r} \frac{\mathrm{d}}{\mathrm{d} r}+\frac{2 \mu}{\hbar^{2}}\left(E_{n, l}^{\mathrm{D}}-V(r)\right)\right.} \\
& \left.\quad-\frac{l(l+D-2)}{r^{2}}\right] R_{n, l}(r)=0, \quad 0 \leq r \leq \infty,
\end{aligned}
$$




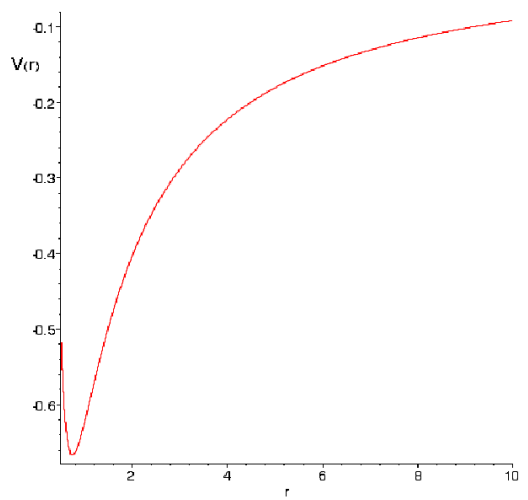

Fig. 1. Manning-Rosen potential vs. $r$.

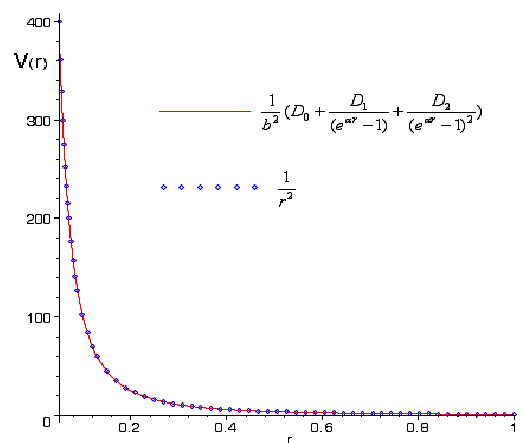

Fig. 2. The potential and its approximation.

where $l$ is the angular momentum quantum number. To get rid of the first order derivative, we apply [5]:

$$
U_{n, l}(r)=r^{\frac{D-1}{2}} R_{n, l}(r),
$$

which results in

$$
\begin{gathered}
\frac{\mathrm{d}^{2} U_{n, l}(r)}{\mathrm{d} r^{2}}+\frac{2 \mu}{\hbar^{2}}\left[E_{n, l}^{\mathrm{D}}-\frac{V_{1}}{\left(\mathrm{e}^{\alpha r}-1\right)^{2}}-\frac{V_{2}}{\mathrm{e}^{\alpha r}-1}\right. \\
\left.-\frac{\hbar^{2}(D+2 l-1)(D+2 l-3)}{8 \mu r^{2}}\right] U_{n, l}(r)=0 .
\end{gathered}
$$

According to the Pekeris approximation, we shall replace inverse square term by $[31,32]$

$$
\frac{1}{r^{2}} \sim \alpha^{2}\left(D_{0}+\frac{D_{1}}{\mathrm{e}^{\alpha r}-1}+\frac{D_{2}}{\left(\mathrm{e}^{\alpha r}-1\right)^{2}}\right),
$$

where $D_{0}=1 / 12, D_{1}=1$ and $D_{2}=1$. In Fig. 2 we have plotted both sides of relation (6) to reveal the reasonability of the approximation. Substituting Eq. (6) into Eq. (5), we obtain

$$
\begin{aligned}
& \frac{\mathrm{d}^{2} U_{n, l}(r)}{\mathrm{d} r^{2}}+\frac{\eta_{2}}{\mathrm{e}^{\alpha r}-1} U_{n, l}(r)+\frac{-\eta_{1}}{\left(\mathrm{e}^{\alpha r}-1\right)^{2}} U_{n, l}(r) \\
& =\left[-\frac{2 \mu}{\hbar^{2}} E_{n, l}^{\mathrm{D}}+\frac{(D+2 l-1)(D+2 l-3)}{4} \alpha^{2} D_{0}\right] \\
& \times U_{n, l}(r),
\end{aligned}
$$

$$
\begin{aligned}
& \eta_{1}=\frac{2 \mu}{\hbar^{2}} V_{1}+\frac{(D+2 l-1)(D+2 l-3)}{4} \alpha^{2} D_{2}, \\
& \eta_{2}=-\frac{2 \mu}{\hbar^{2}} V_{2}-\frac{(D+2 l-1)(D+2 l-3)}{4} \alpha^{2} D_{1} .
\end{aligned}
$$

For further purposes, we write Eq. (7a) as

$$
\left[-\frac{\mathrm{d}^{2}}{\mathrm{~d} r^{2}}+V_{\mathrm{eff}, n, l}(r)\right] U_{n, l}(r)=\lambda_{n, l} U_{n, l}(r)
$$

with

$$
\begin{aligned}
& V_{\text {eff }}(r)=\frac{\eta_{1}}{\left(\mathrm{e}^{\alpha r}-1\right)^{2}}-\frac{\eta_{2}}{\mathrm{e}^{\alpha r}-1}, \\
& \lambda_{n, l}=\frac{2 \mu}{\hbar^{2}} E_{n, l}^{\mathrm{D}}-\frac{(D+2 l-1)(D+2 l-3)}{4} \alpha^{2} D_{0} .
\end{aligned}
$$

The latter is apparently a Schrödinger-like equation. Based on SUSYQM, the superpotential must be the first ingredient we search for. Our superpotential is [30]:

$$
W(r)=\frac{-A}{\mathrm{e}^{\alpha r}-1}+B,
$$

where

$$
\begin{aligned}
& A=\frac{\alpha+\sqrt{\alpha^{2}+4 \eta_{1}}}{2}, \\
& B=\frac{\left(-A^{2}+\eta_{1}+\eta_{2}\right)}{2 A}, \\
& \lambda_{0, l}=-B^{2},
\end{aligned}
$$

and $A, B>0$. On the other hand, the Hamiltonian of Eq. (7) is constructed from the superpotential via $[3,4]$ :

$$
V_{ \pm}(r)=W^{2}(r) \pm W^{\prime}(r) \text {. }
$$

The ground state wave function $U_{0, l}(r)$ is simply calculated from $[3,4]$

$$
U_{0, l}(r)=N_{0, l} \exp \left(-\int W(r) \mathrm{d} r\right) .
$$

If the shape invariance condition holds, i.e. [3, 4]:

$$
V_{+}\left(a_{0}, r\right)=V_{-}\left(a_{1}, r\right)+R\left(a_{1}\right),
$$

where $a_{1}$ is a new set of parameters uniquely determined from the old set $a_{0}$ via the mapping $F: a_{0} \mapsto a_{1}=F\left(a_{0}\right)$ and the residual term $R\left(a_{1}\right)$ dose not include the variable $r$, we simply have $[3,4]$ :

$$
\begin{aligned}
& H_{s}=-\frac{\partial^{2}}{\partial x^{2}}+V_{-}\left(a_{s}, x\right)+E_{s}, \\
& H_{s} \phi_{n-s}^{-}\left(a_{s}, x\right)=E_{n} \phi_{n-s}^{-}\left(a_{s}, x\right), \quad n \geq s, \\
& \phi_{n-s}^{-}\left(a_{s}, x\right)=\frac{A^{\dagger}}{\left[E_{n}-E_{s}\right]^{1 / 2}} \phi_{n-(s+1)}^{-}\left(a_{s+1}, x\right), \\
& A_{s}^{\dagger}=-\frac{\partial}{\partial x}+\Phi\left(a_{s}, x\right), \\
& E_{n}=\sum_{k=1}^{n} R\left(a_{k}\right) .
\end{aligned}
$$

In our case, the partner potentials are

$$
\begin{aligned}
& V_{+}(r)=\frac{A(A+\alpha) \mathrm{e}^{\alpha r}}{\left(\mathrm{e}^{\alpha r}-1\right)^{2}}-\frac{\eta_{1}+\eta_{2}}{\mathrm{e}^{\alpha r}-1} \\
& +\left(\frac{-A^{2}+\eta_{1}+\eta_{2}}{2 A}\right)^{2}
\end{aligned}
$$

and 


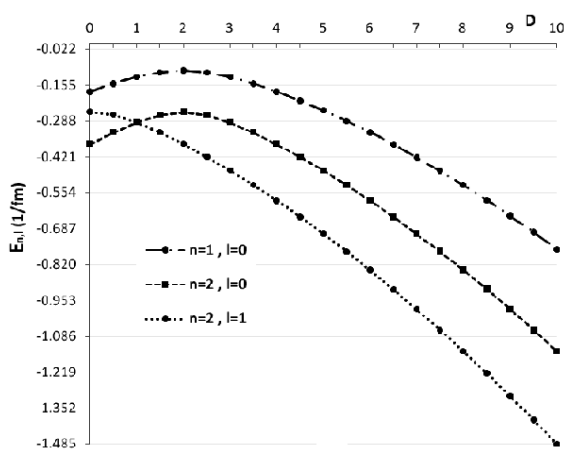

Fig. 3. Energy vs. $D$ for $n=1,2$ and various $l$ 's. For $\mu=1, \hbar=1, \alpha=0.5, \alpha^{\prime}=1.2, b=0.2$.

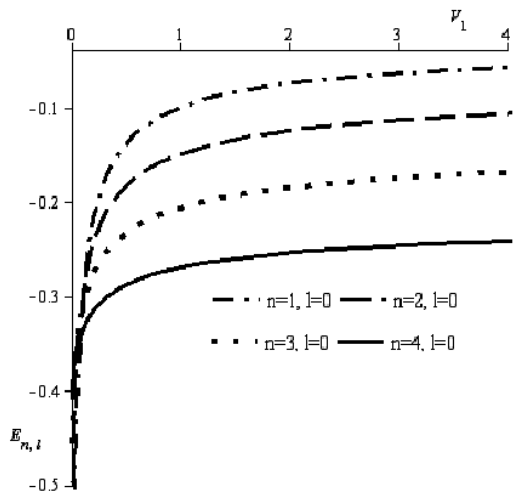

Fig. 4. Energy vs. $V_{1}$.

$$
\begin{aligned}
& V_{-}(r)=\frac{A(A-\alpha) \mathrm{e}^{\alpha r}}{\left(\mathrm{e}^{\alpha r}-1\right)^{2}}-\frac{\eta_{1}+\eta_{2}}{\mathrm{e}^{\alpha r}-1} \\
& +\left(\frac{-A^{2}+\eta_{1}+\eta_{2}}{2 A}\right)^{2} .
\end{aligned}
$$

As we see, the partner Hamiltonians are shape invariant via a mapping of the form $A \rightarrow A+\alpha$. The energy eigenvalues of Hamiltonian

$$
H_{-}(r)=-\frac{\mathrm{d}^{2}}{\mathrm{~d} r^{2}}+V_{-}(r)
$$

are given by

$$
\begin{aligned}
\lambda_{0, l}^{(-)} & =0, \\
\lambda_{n, l}^{(-)} & =\sum_{k=1}^{n} R\left(a_{k}\right)=\left(\frac{-A^{2}+\eta_{1}+\eta_{2}}{2 A}\right)^{2} \\
- & {\left[\frac{-(A+n \alpha)^{2}+\eta_{1}+\eta_{2}}{2(A+n \alpha)}\right]^{2} . }
\end{aligned}
$$

So the complete energy spectrum is given by

$$
\lambda_{n, l}=\lambda_{n, l}^{(-)}+\lambda_{0, l}=-\left[\frac{-(A+n \alpha)^{2}+\eta_{1}+\eta_{2}}{2(A+n \alpha)}\right]^{2} .
$$

Comparing Eq. (20) with Eq. (9b), we obtain the spectrum as

$$
E_{n, l}^{\mathrm{D}}=\frac{\hbar^{2}}{2 \mu}\left\{\frac{(D+2 l-1)(D+2 l-3)}{4} \alpha^{2} D_{0}\right.
$$

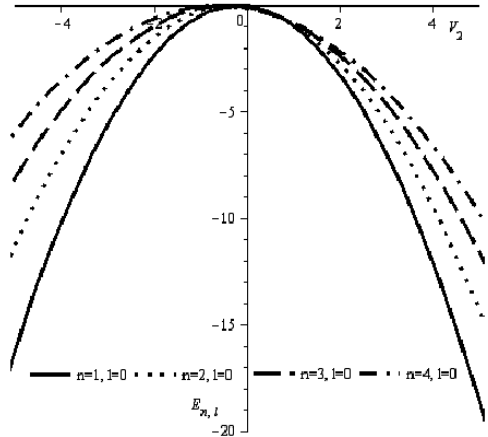

Fig. 5. Energy vs. $V_{2}$.

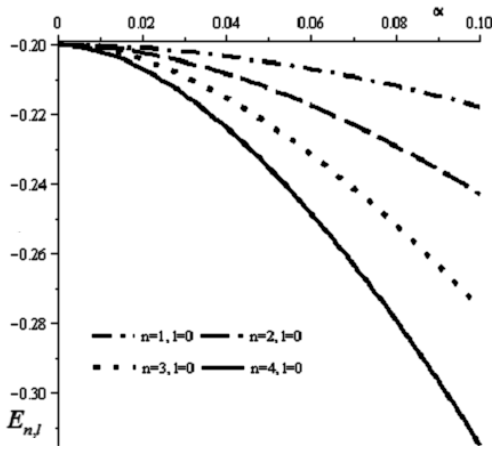

Fig. 6. Energy vs. $\alpha$.

$$
\left.-\left[\frac{-(A+n \alpha)^{2}+\eta_{1}+\eta_{2}}{2(A+n \alpha)}\right]^{2}\right\} .
$$

Figure 3 represents the energy behavior for $n=1,2$ and 3 . In addition, to provide a better insight about the role of the parameters engaged, we have plotted energy vs. $V_{1}, V_{2}$ and $\alpha$ in Figs. $4-6$.

\section{Some expectation values for the Manning-Rosen potential in $D$-dimensions}

We now calculate some expectation values of the Manning-Rosen potential using the Hellmann-Feynman theorem (HFT) [33-35]. Suppose the Hamiltonian $H$ for a particular quantum system is a function of some parameters $q$, and let $E_{n, l}(q)$ and $U_{n, l}(q)$ be the eigenvalues and eigenfunctions of $H$, respectively. According to the HFT theorem,

$$
\frac{\partial E_{n, l}(q)}{\partial q}=\left\langle U_{n, l}(q)\left|\frac{\partial H(q)}{\partial q}\right| U_{n, l}(q)\right\rangle,
$$

bearing in mind that our effective Hamiltonian was

$$
\begin{aligned}
H & =-\frac{\hbar^{2}}{2 \mu} \frac{\mathrm{d}^{2}}{\mathrm{~d} r^{2}}+\frac{\hbar^{2}}{2 \mu} \frac{(D+2 l-1)(D+2 l-3)}{4 r^{2}} \\
& +\frac{V_{1}}{\left(\mathrm{e}^{\alpha r}-1\right)^{2}}+\frac{V_{2}}{\mathrm{e}^{\alpha r}-1} .
\end{aligned}
$$

In order to calculate $\left\langle\left(\mathrm{e}^{\alpha r}-1\right)^{-2}\right\rangle$, we set $q=V_{1}$ such 


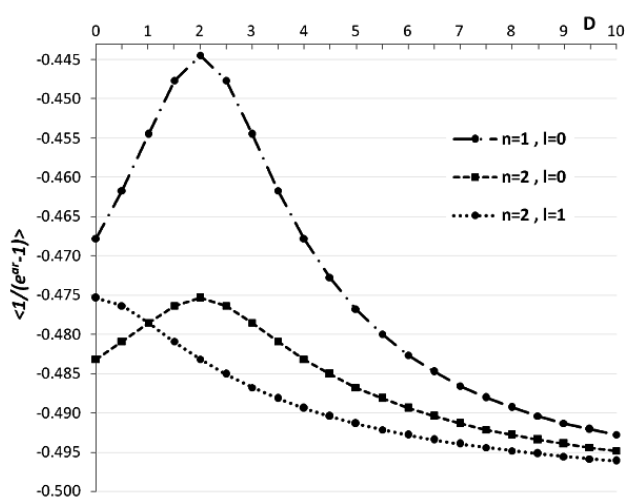

Fig. 7. $\left\langle\left(\mathrm{e}^{\alpha r}-1\right)^{-1}\right\rangle$ vs. $D$ for $n=1$ and 2. For $\mu=1$, $\hbar=1, \alpha=0.5, \alpha^{\prime}=1.2, b=0.2$.

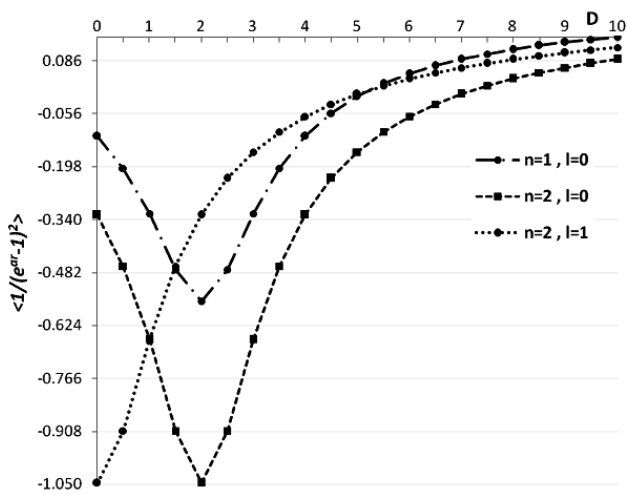

Fig. 8. $\left\langle\left(\mathrm{e}^{\alpha r}-1\right)^{-2}\right\rangle$ vs. $D$ for $n=1$ and 2. For $\mu=1$, $\hbar=1, \alpha=0.5, \alpha^{\prime}=1.2, b=0.2$.

that [35]:

$$
\begin{aligned}
& \frac{\partial E_{n, l}}{\partial V_{1}}=\left\langle U_{n, l}\left(V_{1}\right)\left|\frac{\partial H\left(V_{1}\right)}{\partial V_{1}}\right| U_{n, l}\left(V_{1}\right)\right\rangle \\
& \quad=\left\langle\left(\mathrm{e}^{\alpha r}-1\right)^{-2}\right\rangle .
\end{aligned}
$$

In order to calculate $\left\langle\left(\mathrm{e}^{\alpha r}-1\right)^{-1}\right\rangle$, we set $q=V_{2}$ such that

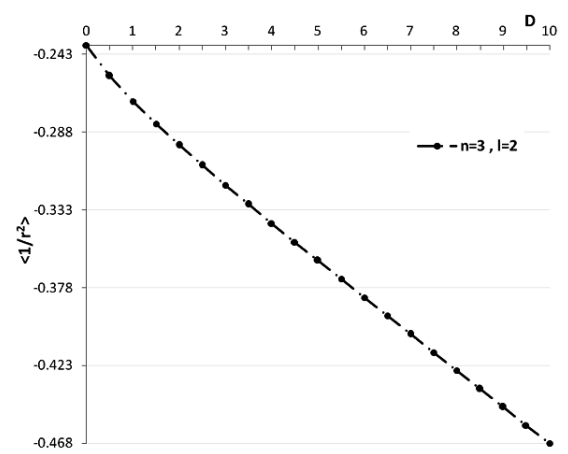

Fig. 9. $\left\langle r^{-2}\right\rangle$ vs. $D$ for $n=3$. For $\mu=1, \hbar=1$, $\alpha=0.5, \alpha^{\prime}=1.2, b=0.2$.

$$
\begin{aligned}
& \frac{\partial E_{n, l}}{\partial V_{2}}=\left\langle U_{n, l}\left(V_{2}\right)\left|\frac{\partial H\left(V_{2}\right)}{\partial V_{2}}\right| U_{n, l}\left(V_{2}\right)\right\rangle \\
& \quad=\left\langle\left(\mathrm{e}^{\alpha r}-1\right)^{-1}\right\rangle .
\end{aligned}
$$

In Figs. 7-9 we have plotted $\left\langle\left(\mathrm{e}^{\alpha r}-1\right)^{-1}\right\rangle,\left\langle\left(\mathrm{e}^{\alpha r}-1\right)^{-2}\right\rangle$ and $\left\langle r^{-2}\right\rangle$ versus $D$ for $U_{1,0}, U_{2,0}$ and $U_{2,1}$.

\section{Conclusion}

We obtained solutions of the $D$-dimensional Schrödinger equation with the Manning-Rosen potential within the framework SUSQM after applying an approximation to the centrifugal term. We calculated the most useful expectation values of the system using the Hellmann-Feynman theorem which reveal the role of engaged terms. Instead of focusing on a particular system, which requires an extensive phenomenological survey (also due to the lack of sufficient experimental data) we included various figures which indicate the role of various terms in the spectrum of the system. We see that the energy is symmetric with respect to $D=2$ for $D=1$ and $D=3$. While the energy magnitude shows an increasing behavior for increasing $V_{2}$, it is decreasing for increasing $V_{1}$. In addition, we observe the detailed behavior of energy in Fig. 6. Our results can be directly used to various systems after proper fits done.

\section{References}

[1] A.F. Nikiforov, V.B. Uvarov, Special Functions of Mathematical Physics, Birkhäuser, Basel 1988.

[2] A. Arda, R. Sever, C. Tezcan, Phys. Scr. 79, 015006 (2009).

[3] F. Cooper, A. Khare, U. Sukhatme, Phys. Rep. 251, 267 (1995).

[4] G. Junker, Supersymmetric Methods in Quantum and Statistical Physics, Springer-Verlag, New York 1996.

[5] S.-H. Dong, Wave Equations in Higher Dimensions, Springer-Verlag, New York 2011.

[6] H. Ciftci, R.L. Hall, N. Saad, J. Phys. A, Math. Theor. 36, 11807 (2003).

[7] Y.P. Varshni, Phys. Rev. A 36, 3009 (1987).

[8] Z.Q. Ma, B.W. Zhu, Eur. Phys. Lett. 69, 685 (2005).

[9] J.C. Slater, Phys. Rev. 81, 385 (1951).

[10] P.M. Stevenson, Phys. Rev. D 23, 2916 (1981).

[11] H. Hassanabadi, B.H. Yazarloo, S. Zarrinkamar, A.A. Rajabi, Phys. Rev. C 84, 064003 (2011).

[12] D. Agboola, Y. Zhang, J. Math. Phys. 53, 042101 (2012).

[13] S.H. Dong, Z. Ma, G. Espozito, Found. Phys. Lett. 12, 465 (1999).

[14] M.F. Manning, N. Rosen, Phys. Rev. 44, 943 (1933).

[15] A.A. Khelashvili, Theor. Math. Phys. 51, 447 (1982).

[16] J.N. Ginocchio, A. Leviatan, Phys. Lett. B 425, 1 (1998).

[17] M.V. Zhukov, B.V. Danilin, D.V. Fedorov, J.S. Vaagen, F.A. Gareev, J. Bang, Phys. Lett. B 265, 19 (1991). 
[18] A. Arda, nad R. Sever, J. Math. Chem., 2012, DOI: 10.1007/s10910-012-0011-0.

[19] A. Rahan, F.H. Stillinger, H.L. Lemberg, J. Chem. Phys. 63, 5223 (1975).

[20] M.F. Manning, N. Rosen, Phys. Rev. 44, 943 (1933).

[21] R.J. Le Roy, R.B. Bernstein, J. Chem. Phys. 52, 3869 (1970).

[22] J. Cai, P. Cai, A. Inomata, Phys. Rev. A 34, 4621 (1986).

[23] E. Romera, P. Sánchez-Moreno, J.S. Dehesa, J. Math Phys. 47, 103504 (2006).

[24] B. Durand, L. Durand, Phys. Rev. D 23, 1092 (1981).

[25] R. Dutt, K. Chowdhury, Y.P. Varshni, J. Phys. A, Math. Gen. 18, 1379 (1985).

[26] C.-S. Jia, J.Y. Wang, S. He, L.-T. Sun, J. Phys. A, Math. Gen. 33, 6993 (2000).
[27] J.A. Olson, D.A. Micha, J. Chem. Phys. 68, 4352 (1978).

[28] G.F. Wei, S.H. Dong, Phys. Lett. B 686, 288 (2010).

[29] G.F. Wei, Z.Z. Zhen, S.H. Dong, Central Europ. J. Phys. 7, 175 (2009).

[30] G. Chen, Z.-D. Chen, Z.-M. Lou, Phys. Lett. A 331 , 374 (2004).

[31] W.C. Qiang, S.H. Dong, Phys. Lett. A 368, 13 (2007).

[32] S.M. Ikhdair, Phys. Scr. 83, 015010 (2011).

[33] G. Hellmann, Introduction to Quantum Chemistry, Deuticke, Leipzig 1937, (in German).

[34] R.P. Feynman, Phys. Rev. 56, 340 (1939).

[35] D. Agboola, Phys. Scr. 80, 065304 (2009). 